\title{
Função Diastólica e Biomarcadores de Participantes de Caminhada de Longa Distância
}

\author{
Diastolic Function and Biomarkers of Long-Distance Walking Participants
}

Maicon Borges Euzebio, ${ }^{1,2}$ Priscila Valverde de O. Vitorino, ${ }^{2 \oplus}$ Watila Moura Sousa, ${ }^{1 \oplus}$ Milena Andrade Melo, ${ }^{3}$ Sérgio Henrique Nascente Costa, ${ }^{2,6}$ Ana Luiza Lima Sousa, ${ }^{3,4}$ Thiago de Souza Veiga Jardim, ${ }^{3,4}$ Ana Carolina Arantes, ${ }^{3,4}$ Paulo Cesar B. Veiga Jardim, ${ }^{5 \bullet}$ Weimar Kunz Sebba Barroso ${ }^{3,4}{ }^{\circledR}$

Universidade Federal de Goiás - Medicina, ${ }^{1}$ Goiânia, GO - Brasil

Pontifícia Universidade Católica de Goiás, ${ }^{2}$ Goiânia, GO - Brasil

Universidade Federal de Goiás - Pós-graduação em Ciências da Saúde, ${ }^{3}$ Goiânia, GO - Brasil

Universidade Federal de Goiás - Liga de Hipertensão Arterial, ${ }^{4}$ Goiânia, GO - Brasil

Universidade Federal de Goiás - Cardiologia, ${ }^{5}$ Goiânia, GO - Brasil

Faculdade da Polícia Militar do Estado de Goiás, ${ }^{6}$ Goiânia, GO - Brasil

\section{Resumo}

Fundamento: Os efeitos da caminhada de longa distância sobre o sistema cardiovascular são pouco estudados.

Objetivos: O objetivo geral deste estudo foi verificar esses efeitos sobre o comportamento da função diastólica e dos biomarcadores cardíacos CK-MB (massa), troponina T e NT-proBNP em atletas amadores.

Método: Este estudo longitudinal realizado em 2015 avaliou os participantes nas 5 etapas seguintes: A0 (basal) antes de iniciar o percurso, e as demais, A1, A2, A3 e A4 ao final de cada dia, totalizando 244,7 km. Em todas as etapas foram dosados os biomarcadores NT-proBNP, CK-MB (massa) e troponina T. Realizou-se ecocardiograma para análise das ondas $E$, A e E'. Adotado $p<0,05$ como significativo.

Resultados: Foram avaliados 25 participantes com média de idade de $46 \pm 10,5$ anos e índice de massa corporal de $20,2 \pm 2,3 \mathrm{~kg} / \mathrm{m} 2$. Encontrou-se aumentos dos valores de NT-proBNP de A0 para A1, A2, A3 e A4 (p < 0,001), CK-MB (massa) de A0 para A2 (p < 0,001) e da onda $E^{\prime}$ de A0 para A1, A2, A3 e A4 (p < 0,001). Foram identificadas correlações entre os seguintes: CK-MB (massa) e troponina $T(A 1: r=0,524, p=0,010 ; A 4: r=0,413, p=0,044)$; CK-MB (massa) e NT-proBNP (A4: $r=0,539, p=0,006)$; e E/A e $E^{\prime}(A 0: r=0,603, p<0,001$; $A 1$ : $r=0,639, p<0,001 ; A 4: r=0,593$, $p=0,002)$; e correlação negativa entre CK-MB (massa) com $E / A(A 1: r=-0,428, p=0,041)$.

Conclusão: Os efeitos da atividade física intensa, prolongada e intercalada foram verificados a partir das variações significativas no comportamento da CK-MB (massa), NT-proBNP e E'. Apesar das alterações encontradas, não houve critérios sugestivos de dano ao miocárdio. (Arq Bras Cardiol. 2020; 115(4):620-627)

Palavras-chave: Caminhada; Biomarcadores Biológicos; Pressão Sanguínea; Troponina T; Peptídeo Natriurético Cerebral; Atletas; Ecocardiografia Doppler/métodos.

\begin{abstract}
Background: The effects of long-distance walking on the cardiovascular system have been little studied.

Objectives: The general objective of this study was to verify these effects on the behavior of diastolic function and the cardiac biomarkers CK-MB (mass), troponin T, and NT-proBNP, in amateur athletes.

Method: This longitudinal study, conducted in 2015, evaluated participants during the following 5 stages: EO (baseline) before starting the trajectory and the others, E1, E2, E3, and E4, at the end of each day, totaling $244.7 \mathrm{~km}$. At all stages, the biomarkers NT-proBNP, CK-MB (mass), and troponin $T$ were measured. Echocardiogram was performed to analyze the $E, A$ and $E^{\prime}$ waves. $P<0.05$ was adopted as significant.

Results: The study evaluated 25 participants, with an average age of $46 \pm 10.5$ years and body mass index of $20.2 \pm 2.3 \mathrm{~kg} / \mathrm{m} 2$. Increased values were found for NT-proBNP from EO to E1, E2, E3, and E4 ( $<<0.001)$, CK-MB (mass) from EO to E2 ( $p<0.001)$, and $E^{\prime}$ wave from EO to E1, E2, E3, and E4 ( $p<0.001)$. Positive correlations were identified between the following: CK-MB (mass) and troponin $T$ (E1: $r=0.524$, $p=0.010 ; E 4: r=0.413, p=0.044) ; C K-M B$ (mass) and NT-proBNP (E4: $r=0.539, p=0.006) ;$ and $E / A$ and $E^{\prime}(E 0: r=0.603, p<0.001 ; E 1$ : $r=0.639, p<0.001 ; E 4: r=0.593, p=0.002$ ). A negative correlation was found between CK-MB (mass) and E/A $(E 1: r=-0.428, p=0.041)$.
\end{abstract}

Correspondência: Maicon Borges Euzebio •

Universidade Federal de Goiás - Medicina - Programa de Pós-Graduação em Ciências da Saúde - R. 235, s/n. CEP 74605-050, Setor Leste Universitário,

Goiânia, GO - Brasil

Artigo recebido em 26/04/2019, revisado em 07/08/2019, aceito em 10/09/2019

E-mail: maiconborges@gmail.com, sebbabarroso@gmail.com

DOI: https://doi.org/10.36660/abc.20190271 
Conclusion: The effects of intense, prolonged, and interspersed physical activity were verified based on significant variations in the behavior of CK-MB (mass), NT-proBNP, and the $E^{\prime}$ wave. Notwithstanding the alterations found, there were no criteria suggestive of myocardial damage (Arq Bras Cardiol. 2020; 115(4):620-627)

Keywords: Walking; Biomarkers, Biological; Blood Pressure; Troponin-T; Natriuretic Peptide Brain; Athletes; Echocardiography, Doppler/methods.

Full texts in English - http://www.arquivosonline.com.br

\section{Introdução}

O exercício físico é fundamental para a manutenção da saúde geral e prevenção de doenças. ${ }^{1}$ Entretanto, a intensidade, duração e frequência são fatores que separam os benefícios dos prejuízos no organismo humano. Evidências recentes questionam se a demanda fisiológica para manter um débito cardíaco elevado durante um período prolongado de exercício pode resultar em comprometimento transitório das funções cardíacas. ${ }^{2}$

Os efeitos agudos do exercício físico na função diastólica e até mesmo as variações de disfunção diastólica estão relacionadas indiretamente à estimulação persistente, dependendo também da intensidade e duração. Essas adaptações, mesmo em curto prazo, podem produzir diminuição da função cardíaca, fato esse conhecido como fadiga cardíaca. ${ }^{3}$

As enzimas cardíacas creatina quinase fração $M B$ (CK-MB, creatine kinase-MB), troponina $\mathrm{T}$ cardíaca e do aminoácido precursor $\mathrm{N}$-terminal do peptídeo natriurético cerebral tipo B (NT-proBNP, N-terminal pro B-type natriuretic peptide) são biomarcadores importantes para a avaliação da existência de lesões miocárdicas e da função diastólica. ${ }^{4}$

Níveis de CK-MB também podem elevar em estados de rabdomiólise e até de apoplexia, possuindo dessa forma uma sensibilidade variável. ${ }^{5}$ A troponina T é o marcador preferencial de lesões miocárdicas, considerado como padrão ouro. ${ }^{6}$

O NT-proBNP está associado direta e paralelamente com as concentrações do peptídeo natriurético cerebral (BNP brain natriuretic peptide). Ele pode ser utilizado para avaliação diagnóstica e prognóstica da insuficiência ventricular esquerda e também se eleva em condições que induzem disfunção diastólica. Pouco se conhece sobre o comportamento desses biomarcadores e especialmente do significado prognóstico em indivíduos saudáveis submetidos a stress físico intenso.?

No entanto, poucas pesquisas avaliaram o efeito da caminhada de longa distância, de moderada a alta intensidade, no sistema cardiovascular a partir da avaliação da função diastólica e dos biomarcadores cardíacos. Este foi o primeiro estudo a avaliar a função diastólica e os biomarcadores cardíacos nesse tipo de exercício. Dessa forma o objetivo do presente estudo foi verificar, em atletas amadores, os efeitos de caminhada de longa distância, de moderada a alta intensidade, sobre o comportamento da função diastólica e dos biomarcadores cardíacos CK-MB (massa), troponina T e NT-proBNP.

\section{Métodos}

Este foi um estudo longitudinal, realizado durante caminhada de longa distância no ano de 2015 - 24ạ Caminhada Ecológica de Goiás. Inicialmente, cerca de duzentas pessoas se inscreveram pela internet para participar. Os candidatos participaram de uma seletiva, na qual percorreram $56 \mathrm{~km}$, divididos em dois dias (28 km cada), os quais deveriam ser cumpridos em até três horas e dez minutos para os homens e três horas e 30 minutos para as mulheres. Os classificados de acordo com o melhor tempo de cada faixa etária foram selecionados, totalizando 29 participantes, sendo 25 do sexo masculino e quatro do sexo feminino. As mulheres foram retiradas da análise por não completarem diariamente o percurso previamente estabelecido.

O projeto de pesquisa foi aprovado pelo Comitê de Ética em Pesquisa da Pontifícia Universidade Católica de Goiás sob parecer de número 1.107.021. Os indivíduos aprovados na seletiva foram convidados a participar do estudo. Após o aceite, aplicou-se o Termo de Consentimento Livre e Esclarecido e foi realizada a avaliação inicial (A0) em uma unidade de avaliação cardiovascular. As outras avaliações aconteceram 34 dias após A0 ao longo do trajeto de 244,7 $\mathrm{km}$, ao final de cada um dos dias e nos locais de repouso de cada cidade do percurso no período de 21 a 24 de julho de 2015 sendo cognominadas de A1, A2, A3 e A4 (avaliação do $1^{\circ}$ dia $2^{\circ}$ dia $3^{\circ}$ dia e $4^{\circ}$ - dia). Não foram realizadas coletas de dados no último dia do evento pois os participantes foram dispensados para retornarem às suas cidades.

A dominância do relevo foi dada em subida, em descida e nivelado, conforme cada dia do percurso. Essas informações foram obtidas de um estudo anterior que já havia avaliado esse percurso. ${ }^{8}$ A declividade do traçado do terreno foi obtida por meio do software ArCGIS 10.3 pela ferramenta de declividade (slope, arctoolbox), a partir das imagens Shuttle Radar Topography Mission (SRTM) fornecidas pelo serviço geológico americano de três arcseg de tamanho de pixel. Para a monitorização meteorológica dos dias do percurso (A1 a A4) foram utilizados os dados da estação Goiás denominada oficialmente pelo Instituto Nacional de Meteorologia - INMET de A014.

\section{Organização da Coleta}

O ambiente de pesquisa foi subdividido em estações, uma para cada avaliação. As avaliações foram compostas por anamnese (somente em A0); ecocardiografia e coleta de sangue para avaliação de biomarcadores em todas as etapas. As coletas dos dados ecocardiográficos e biomarcadores começavam por volta das 18 horas (30 a 120 minutos após o final da caminhada) de cada dia do percurso. Dessa forma, quando os participantes chegavam nos locais de repouso, já haviam sido preparadas estações de pesquisa iniciando o fluxo pela identificação dos participantes e depois pela coleta de sangue e avaliação ecocardiográfica, sendo posteriormente dispensados para voltarem a rotina do evento. Não foi possível realizar coleta pela manhã pois os participantes levantavam 
às 04 horas da madrugada e isso poderia prejudicá-los por interferir nos seus períodos de descanso e recuperação.

A anamnese foi organizada pelos pesquisadores em formato de entrevista com questões referentes a dados pessoais como idade (em anos), sexo (masculino e feminino), profissão e estado civil; antecedentes pessoais e familiares: hipertensão arterial, diabetes e dislipidemia; uso de medicamento(s); tabagismo (atual/anterior); quantidade de cigarros por dia para fumantes; prática de exercício físico (frequência semanal, duração diária e tempo de prática); presença de problema ortopédico que dificultasse a realização de exercícios.

\section{Procedimentos para a Realização dos Exames de Biomarcadores Sanguíneos e Ecocardiograma}

Foi utilizada a técnica para a coleta de sangue por meio de acesso venoso periférico em fossa cubital esquerda. ${ }^{9}$ Foram extraídos cerca de $5 \mathrm{ml}$ de sangue de cada participante. O sangue foi centrifugado no local da coleta a $3.000 \mathrm{rpm}$ durante 10 minutos para a obtenção do soro/plasma. O soro foi colhido em tubos de amostra com gel separador. As amostras foram prontamente armazenadas e congeladas a $-20 \stackrel{\circ}{ } \mathrm{C}$ em congelador específico nas estações de coleta. ${ }^{10}$ As amostras foram encaminhadas para um laboratório credenciado para serem analisadas. Para a realização dos exames dos biomarcadores foram utilizados kits com registro na Agência Nacional de Vigilância Sanitária (ANVISA). Os valores de CKMB (massa), troponina T e NT-proBNP foram obtidos a partir da técnica imunoensaio de electroquimioluminescência. ${ }^{11}$ Os ensaios foram realizados pelo sistema COBAS® - Modular Analytics E170 a partir dos kits seguintes: CK-MB STAT, Troponin T hs e proBNP II respectivamente. Para assegurar a correta execução do ensaio, foram cumpridas todas as instruções fornecidas no documento guia para o analisador. Todos os exames foram processados por biomédico. Foram utilizadas as seguintes unidades de medidas: $\mathrm{pg} / \mathrm{ml}$ para NTproBNP e para troponina T e $\mathrm{ng} / \mathrm{ml}$ para CK- MB (massa). ${ }^{11}$

\section{Ecocardiografia}

O ecocardiograma foi realizado individualmente em todas as etapas da coleta. Em A0 ao longo do dia e, de A1 até A4 por volta das 18 horas, após a chegada dos participantes nas estações de pesquisa. Todos os exames foram realizados pelo mesmo ecocardiografista. Foi realizado ecocardiograma uni e bidimensional com Doppler colorido, utilizando-se o aparelho portátil Philips CX50 ${ }^{8}$, com transdutor eletrônico de $2-5 \mathrm{MHz}$ de frequência. A técnica e os padrões de referência utilizados foram aqueles preconizados pela American Society of Echocardiography. ${ }^{12}$ Foi utilizado o mesmo ângulo de incidência para análise transmitral, procurando-se alinhar o feixe de ultrassom o mais paralelo possível ao fluxo do Doppler colorido. Os parâmetros utilizados para a análise da disfunção diastólica, com suas respectivas unidades de medidas, foram, para fluxo mitral, E em cm/s, A em cm/s e a relação E/A. Para avaliação tecidual foi $\mathrm{E}^{\prime} \mathrm{em} \mathrm{cm} / \mathrm{s}^{13}$

\section{Análise Estatística}

Os dados foram analisados por estatística descritiva com frequências absolutas e relativas, médias, desvio padrão e intervalo de confiança. Para testar a normalidade da distribuição dos dados das variáveis foi utilizado o teste de Shapiro-Wilk. Para as comparações das variáveis referentes aos biomarcadores cardíacos entre os dias da caminhada e referentes aos parâmetros ecocardiográficos, foi realizado o teste de ANOVA para medidas repetidas, seguido do post hoc de Bonferroni. Para as correlações foram utilizados os testes de Pearson ou Spearman. Adotou-se o valor de $p<0,05$ para significância estatística. Para realizar a análise estatística foi utilizado o software Stata, versão 14.

\section{Resultados}

\section{Características da Amostra}

Foram avaliados 25 homens, média de idade de $46 \pm 10,5$ anos e índice de massa corporal de 20,2 $\pm 2,3 \mathrm{~kg} / \mathrm{m}^{2}$ (Tabela 1). Quatro (16\%) participantes utilizavam medicamentos que não influenciaram nas variáveis analisadas. A distância percorrida em quatro dias foi de 244,7 km e velocidade média de $7,6 \mathrm{~km} / \mathrm{h}$. Todos os participantes caminhavam juntos, em bloco, mantendo portanto a mesma velocidade. O período de descanso noturno diário foi entre seis e sete horas.

Em relação aos exercícios físicos prévios, 24 participantes (96\%) praticavam corrida; um (4\%) ciclismo e quatro $(16 \%)$ musculação. A média de tempo de realização prévia de atividade aeróbia foi de 13,3 anos $(0,5 \pm 40)$ e a média da distância semanal de corrida, que foi a atividade relatada como mais praticada, foi de $64 \mathrm{~km}$.

\section{Características Ambientais do Trajeto}

A temperatura durante os dias do percurso variou de 19 a $32{ }^{\circ} \mathrm{C}$ e a umidade relativa de 21 a $77 \%$. Em A1 e A3 o relevo era predominantemente de subida, em A2 de descida (A2) e em A4 plano. As variações mais acentuadas da declividade do terreno foram encontradas nos trechos de A2 e A3, variando de 0 a -15 .

\section{Avaliação dos Biomarcadores e das Ondas de Função Diastólica Durante a Caminhada de Longa Distância}

O NT-proBNP apresentou aumento significativo de A0 para todas as outras avaliações. A CK-MB (massa) teve aumento significativo de A0 para A2 e a troponina $\mathrm{T}$ não apresentou alterações significativas. Quanto a função diastólica, as ondas E e A não sofreram alterações entre os dias da caminhada. A onda E' aumentou de A0 para todos os outros dias (Figura 1).

Foram realizadas correlações entre todos os parâmetros coletados referentes aos biomarcadores e ondas da função diastólica e, evidenciadas somente as correlações significativas. Com relação aos biomarcadores sanguíneos, foram identificadas correlações positivas e moderadas nos seguintes dias de exposição: $A 1$, entre troponina $T$ e CKMB (massa); A4, entre NT-proBNP e CK-MB (massa) e entre troponina T e CK-MB (massa). Na função diastólica, foram identificadas correlações positivas e moderadas entre E/A e $E^{\prime}$ em A0, A1 e A4. Dentre os biomarcadores e as ondas da função diastólica, foi evidenciada correlação negativa e moderada entre CK-MB e E/A em A1 (Tabela 2). 
Tabela 1 - Características sociais, de saúde e de hábitos de vida dos participantes da Caminhada Ecológica de Goiás, Brasil, 2015, $n=25^{*}$

\begin{tabular}{lcc}
\hline Variáveis & $\mathbf{n}$ & $\%$ \\
\hline Faixa etária (anos) & & \\
\hline $19-39$ & 19 & 20 \\
\hline $40-59$ & 1 & 4 \\
\hline$\geq 60$ & & \\
\hline Uso de medicamentos & 21 & 84 \\
\hline Não & 4 & 16 \\
\hline Sim & & \\
\hline Doenças relatadas & 1 & 4 \\
\hline Diabetes mellitus & 1 & 4 \\
\hline Dislipidemia & 2 & 4 \\
\hline Hipotireoidismo & 3 & 12 \\
\hline Problemas ortopédicos &
\end{tabular}

* Número de participantes avaliados

\section{Discussão}

Apesar dos participantes serem considerados atletas amadores, a maioria possuía experiência prévia no percurso e em edições anteriores da Caminhada Ecológica de Goiás com uma média de 7,2 participações; somando-se a isso, o processo seletivo para ingresso proporcionou o surgimento de uma amostra altamente selecionada.

A velocidade de corrida competitiva em meia maratona ou maratona solicita maiores demandas energéticas e metabólicas e amplia a chances de desfechos cardiovasculares, principalmente em indivíduos não condicionados. ${ }^{14}$ No nosso trabalho a velocidade média foi de $7,6 \mathrm{~km} / \mathrm{h}$, bem inferior ao modelo de maratona e meia maratona e, portanto, com menor demanda energética e metabólica dos participantes.

Durante os dias de exposição ao esforço, em comparação aos 30 dias que precederam (A0), ocorreram aumentos significativos dos valores de NT-proBNP, CK-MB (massa) e da onda $E^{\prime}$ (Doppler tecidual). Não observamos variações significativas nos níveis de troponina $\mathrm{T}$ e na relação das ondas de fluxo mitral E/A.

Este aumento também foi verificado em indivíduos saudáveis, adolescentes e adultos, independente do sexo, quando submetidos a exercícios de resistência com retorno aos valores basais nas primeiras 24 horas após o exercício. ${ }^{15,16}$

No presente estudo, encontramos um aumento nos valores das concentrações séricas de NT-proBNP em até cinco vezes após o exercício quando comparado aos níveis basais, dados que corroboram com a literatura. ${ }^{17-19}$ Esses dados reforçam a importância do efeito natriurético no mecanismo de adaptação aguda e subaguda do aparelho cardiovascular ao esforço físico.
Elevações séricas da CK-MB (massa) também foram encontradas em indivíduos após exercícios extenuantes. Em uma pesquisa, realizada nos Estados Unidos com participantes jovens de uma maratona, foram encontrados níveis plasmáticos aumentados e sustentados da CK-MB em até 54 horas pós exposição ao exercício. ${ }^{20,21}$

Indivíduos treinados, que realizam exercício de alta intensidade em práticas esportivas, tendem durante o teste de esforço máximo apresentar de forma aguda maiores elevações séricas da CK-MB do que indivíduos não treinados, sugerindo assim um envolvimento cardíaco e principalmente benigno na natureza desses níveis séricos. ${ }^{22}$

Cientistas também verificaram o efeito do exercício prolongado e das variações de temperatura ambiental no comportamento das creatina quinases e identificaram que quanto mais prolongado o exercício e maior a temperatura ambiental, maiores são as liberações plasmáticas desses biomarcadores. ${ }^{23}$ Quanto mais extenso o trecho percorrido em uma menor quantidade de tempo, maiores as chances de lesões musculares e, portanto, a presença sérica de maior quantidade das creatina quinases. Mais um fato importante é que a força de reação do relevo, nos momentos descida, exercida contra os participantes, pode também ocasionar aumento de lesões musculoesqueléticas, devido à maior fonte de impacto com tendência a um custo metabólico mais acentuado durante o declive. ${ }^{24,25}$

As variações significativas da CK-MB (massa) em nosso estudo foram encontradas somente entre os trechos de $\mathrm{A} 0 \mathrm{e}$ A2. Este aumento pode ter sido relacionado às características do percurso em A2, a saber, predominantemente de descida, com maior variação de declive e com maior extensão quando comparado aos outros dias. O efeito cumulativo da CK-MB (massa) em A1 pode ter influenciado o aumento significativo do valor da CK-MB (massa) em A2.

Existem também comprovações das limitações da avaliação da CK-MB em indivíduos saudáveis durante a prática de exercício físico. Sua especificidade fica prejudicada na presença de processos inflamatórios e de estresse muscular esquelético associado à redução nas primeiras horas de exposição ao esforço pelo aparecimento lentificado desses marcadores no sangue, sendo mais sensível quando há presença de injúria cardíaca. ${ }^{26}$

Em estudos de meia maratona, maratona e ultramaratona de 48 horas, realizados em corredores amadores (indivíduos não atletas), foram encontrados valores elevados de troponina T nas três primeiras horas da pós-exposição com reduções importantes logo a seguir, chegando a níveis basais em no

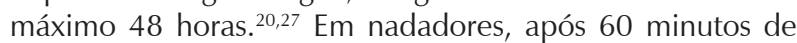
natação, também foi verificado a mesma variação da troponina T que em corredores amadores. ${ }^{16}$

Eijsvogels et al. ${ }^{28}$ avaliaram 82 pessoas durante um trajeto de 30 quilometros/dia durante quatros dias, também identificou que as troponinas tiveram aumento somente no primeiro dia com redução plasmática em todos os outros dias apresentando associação com a velocidade da caminhada. ${ }^{28}$ Uma meta-análise de 45 estudos que avaliaram o comportamento das troponinas e dos BNPs após a exposição a exercícios de resistência observou que os valores plasmáticos 


\section{Artigo Original}
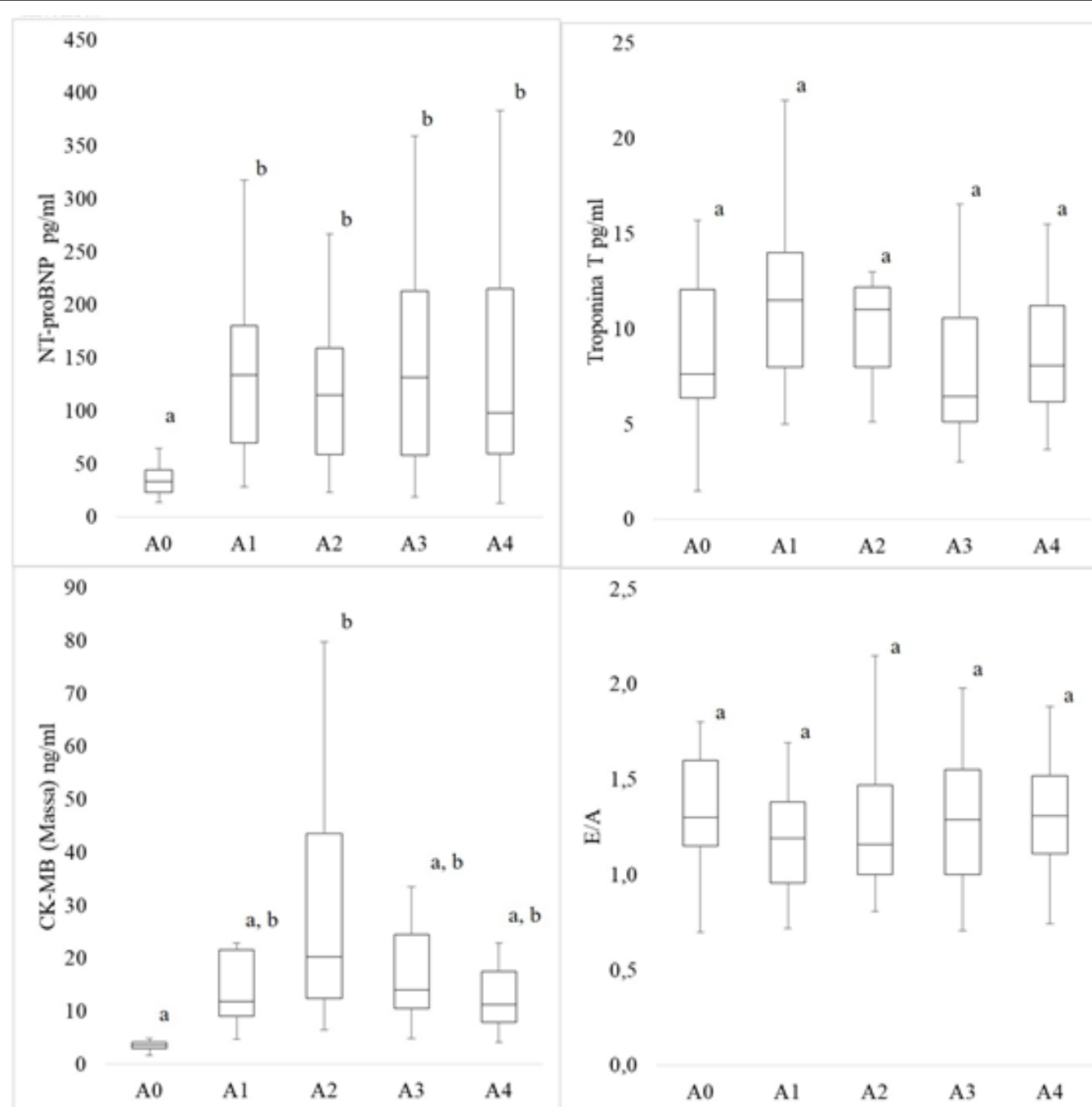

2,5

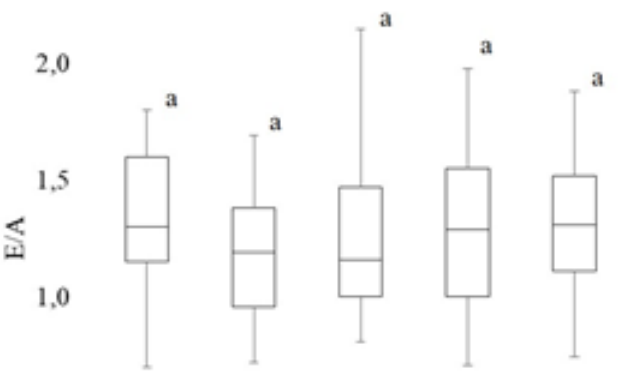

0,5

0,0

$\begin{array}{llllll}0,0 & \text { A0 } & \text { A1 } & \text { A2 } & \text { A3 } & \text { A4 } \\ 20 & & & & & \end{array}$

है

40

20
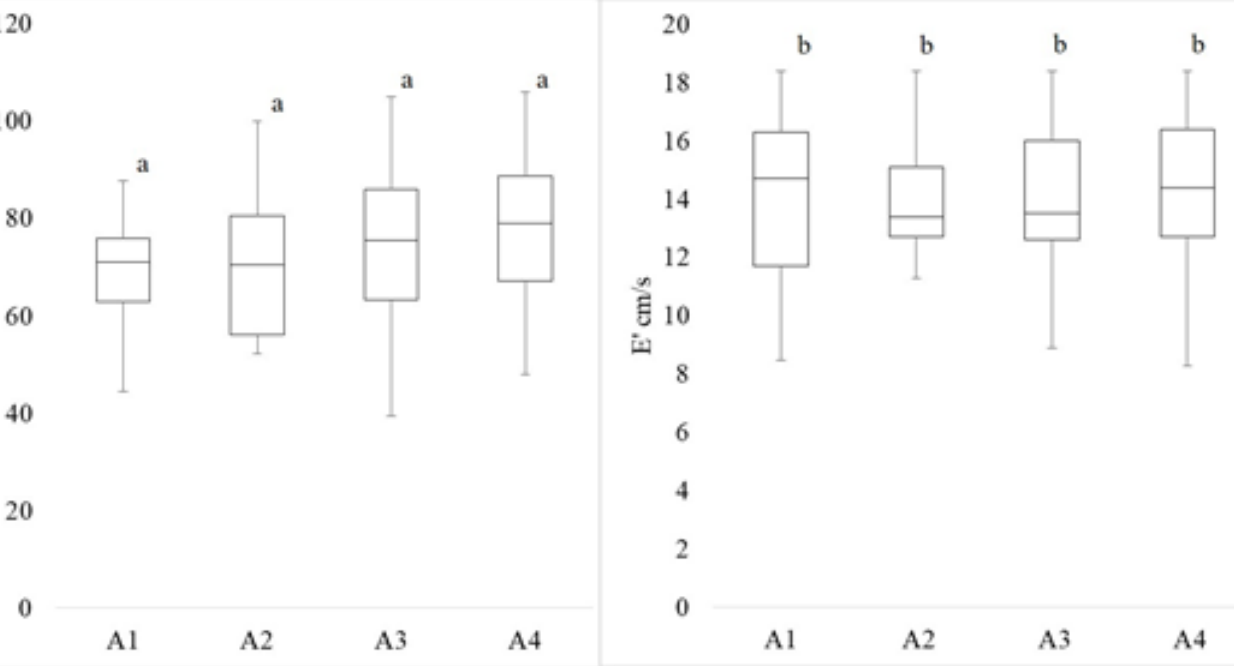

6

4

2

0
Al
A2
A3
A4

A0 (basal); A1 (primeiro dia); A2 (segundo dia); A3 (terceiro dia); A4 (quarto dia). ANOVA e post hoc Bonferroni: letras iguais $\mathrm{p}>0,05$; letras diferentes $\mathrm{p}<0,001$.

Figura 1 - Comparação entre valores de NT-proBNP, Troponina T, CK-MB (Massa), E/A, E e E'obtidos durante a avaliação basal e durante os quatro dias de exposição à atividade fisica de moderada a intensa. 
Tabela 2 - Correlação dos biomarcadores, da função diastólica e dos biomarcadores com a função diastólica de participantes da Caminhada Ecológica de Goiás, Brasil, 2015, $n=25$

\begin{tabular}{|c|c|c|c|}
\hline Avaliação & Parâmetro & $r$ & $\mathrm{p}$ \\
\hline \multirow[t]{2}{*}{$\mathrm{A} 0$} & $\mathrm{E} / \mathrm{A} \times \mathrm{E}^{\prime}$ & 0,603 & $0,001^{*}$ \\
\hline & Troponina T x CK-MB (massa) & 0,524 & $0,010^{* *}$ \\
\hline \multirow[t]{3}{*}{ A1 } & $\mathrm{E} / \mathrm{A} \times \mathrm{E}^{\prime}$ & 0,639 & $0,001^{*}$ \\
\hline & CK-MB (massa) - E/A (seria "x"?) & $-0,428$ & $0,041^{* *}$ \\
\hline & NT-proBNP x CK-MB (massa) & 0,539 & $0,006^{* *}$ \\
\hline \multirow[t]{2}{*}{ A4 } & Troponina T x CK-MB (massa) & 0,413 & $0,044^{* *}$ \\
\hline & $E / A \times E^{\prime}$ & 0,593 & $0,001^{*}$ \\
\hline
\end{tabular}

*Teste de Pearson; ** Teste de Spearman.

elevados das troponinas e BNP durante e após exercício intenso e prolongado são propensos a alterações, podendo representar uma característica aguda frente a exposição ao exercício. ${ }^{29}$ Ainda não está clara a biomecânica da liberação das troponinas induzida pelo exercício físico e se essa realmente reflete um processo fisiológico ou patológico. ${ }^{30}$

A troponina T avaliada no presente estudo não apresentou, durante os dias do percurso, aumento significativo e difere dos resultados encontrados em outros estudos citados. Isto pode estar relacionado aos fatos de os participantes terem percorrido os trajetos em uma velocidade média abaixo dos valores de competição mesmo para corredores amadores; terem alternado corrida com caminhada; e terem sido hidratados rigorosamente durante todo o trajeto. O comportamento da troponina $\mathrm{T}$ foi diferente do geralmente esperado quando relacionado a CK-MB (massa). Como a troponina T tem maior especificidade para dano miocárdico por isquemia é possível que essa seja a razão de menor aumento entre $\mathrm{A} 0 \mathrm{e}$ os demais dias. Este fato pode demonstrar que os danos ao sistema cardiovascular parecem ser mínimos e que essa modalidade de esforço aparenta ser segura. ${ }^{31}$

Em nossa amostra, encontramos aumento significativo da onda $E^{\prime}$ com relação aos valores basais e sem redução da relação das ondas E/A. Esses achados podem estar relacionados às características da população avaliada que era bem condicionada e à intensidade do esforço, concorrendo para uma maior capacidade adaptativa de remodelamento do miocárdio. Outros estudos que fizeram ecocardiografia em diferentes populações tiveram resultados distintos. Não foi encontrada disfunção diastólica ventricular esquerda ou direita em triatletas amadores de média e longa distância. ${ }^{32}$ Já em atletas adultos foram demonstradas alterações do relaxamento miocárdico durante a diástole, ${ }^{33}$ identificada pelo aumento da onda E (fluxo mitral) em comparação com a onda E' (Doppler tecidual). Em exercícios de ultra-resistência houve diminuições significativas na onda $E$ e na relação E/A imediatamente após o exercício. ${ }^{34-36}$ Uma publicação com metodologia mais próxima da que utilizamos avaliou as mudanças da função cardíaca em participantes de uma trilha ecológica e constatou a relação das ondas mitral E/A e Doppler tecidual E' significativamente diminuídas, a partir de $21 \mathrm{~km} .^{37}$

O treinamento regular e aeróbio pode minimizar as alterações agudas da função diastólica frente a solicitação de maiores demandas cardíacas no exercício intenso. Este efeito de treinamento pode exercer um papel fundamental na preservação do preenchimento diastólico em atletas mais velhos..$^{35,38}$

Encontramos correlações positivas entre os seguintes: E/A e $E^{\prime}$; CK-MB (massa) e troponina T; e CK-MB (massa) e NTproBNP. Correlação negativa foi encontrada apenas entre CK-MB (massa) e E/A (relação da velocidade de enchimento ventricular rápido com a velocidade de contração atrial).

Poucos trabalhos realizaram correlação das ondas da função diastólica e das variações séricas dos biomarcadores de lesão cardíaca com as ondas de variação da função diastólica. Sabe-se que em padrões fisiológicos as variações do comportamento do fluxo mitral refletem na mesma direção que as variações do comportamento tecidual ventricular. ${ }^{13}$ Jouffroy et al. ${ }^{37}$ conseguiram encontrar correlação positiva entre E/A e $E^{\prime}$ em participantes amadores de prova de resistência. Os poucos estudos que avaliaram essas correlações têm resultados discordantes. ${ }^{37}$ 
Sabe-se da relação direta do aumento dos BNP(s) com as troponinas, creatina quinases e com as alterações diastólicas. A troponina $\mathrm{T}$ também está fortemente associada às anormalidades de relaxamento ventricular. ${ }^{16,20,39}$ A correlação positiva da CK-MB (massa) com troponina T e NT-proBNP encontrada em nosso trabalho pode estar relacionada ao fato de que mesmo em situações de ausência de isquêmia cardíaca, níveis mínimos desses biomarcadores podem ser liberados na corrente sanguínea e na mesma direção do comportamento da CK-MB (massa).

Por conseguinte, verificamos correlação inversa da CKMB (massa) com a relação das ondas E/A. O motivo que a diminuição de um implica no aumento da outra necessita ser mais estudado já que, talvez, essa correlação possa ser apenas acaso. Podemos especular quanto à possibilidade de que a disfunção diastólica reversível seja um dos possíveis mecanismos de aumento plasmático da CK-MB (massa) com a diminuição da onda $E$ e aumento da onda $A$.

\section{Limitações}

A coleta de dados durante os dias do trajeto teve que se adaptar aos horários disponíveis do evento, podendo ter interferido na avaliação das variações agudas dos biomarcadores cardíacos já que os níveis plasmáticos destes variam conforme o tempo de exposição. Não foi possível, também, realizar coleta matinal para avaliar o comportamento das variáveis após o repouso, já que isso atrapalharia o descanso e o processo de recuperação dos participantes que tinham que acordar as quatro horas da manhã para se preparem e depois percorrerem os trechos estabelecidos. Além disso, a avaliação pós exposição poderia ter ajudado a elucidar melhor questões referentes ao comportamento dos biomarcadores e das ondas da função diastólica como, por exemplo, se houve ou não retorno próximo aos valores basais.

\section{Referências}

1. Piercy KL, Troiano RP. Physical Activity Guidelines for Americans From the US Department of Health and Human Services. Circ Cardiovasc Qual Outcomes. 2018;11(11): e005263.

2. Eijsvogels T, Thompson PD. Are There Clinical Cardiac Complications From Too Much Exercise? Curr Sports Med Rep. 2017;16(1):9-11.

3. Claessen G, La Gerche A. Exercise-induced cardiac fatigue: the need for speed. J Physiol. 2016;594(11):2781-2.

4. Cocking S, Landman T, Benson M, Lord R, Jones H, Gaze D, et al. The impact of remote ischemic preconditioning on cardiac biomarker and functional response to endurance exercise. Scand J Med Sci Sports. 2017;27(10):1061-9.

5. Mythili S, Malathi N. Diagnostic markers of acute myocardial infarction. Biomed Rep. 2015;3(6):743-8.

6. McRae A, Innes G, Graham M, Lang E, Andruchow J, Yang H, et al. Comparative evaluation of 2-hour rapid diagnostic algorithms for acute myocardial infarction using high-sensitivity cardiac Troponin T. Can J Cardiol. 2017 ;33(8):1006-1012.

7. Zabarovskaja S, Hage C, Linde C, Daubert JC, Donal E, Gabrielsen A, et al. Adaptive cardiovascular hormones in a spectrum of heart failure phenotypes. Int J Cardiol. 2015;189:6-11.

\section{Conclusões}

Os efeitos da atividade física intensa, prolongada e intercalada foram verificados a partir das variações significativas no comportamento da CK-MB (massa), NT-proBNP e E'. Vale ressaltar que apesar das alterações encontradas, não houveram critérios demonstrativos de dano ao miocárdio com essa modalidade de esforço em indivíduos treinados.

\section{Contribuição dos Autores}

Concepção e desenho da pesquisa: Euzebio MB, Vitorino PVO, Sousa WM, Sousa ALL, Jardim TSV, Arantes AC, Jardim PCBV, Barroso WKS; Obtenção de dados: Euzebio MB, Sousa WM, Melo MA, Costa SHN, Arantes AC; Análise e interpretação dos dados: Euzebio MB, Vitorino PVO, Sousa WM, Melo MA, Costa SHN, Sousa ALL, Arantes AC, Jardim PCBV, Barroso WKS; Análise estatística: Euzebio MB, Vitorino PVO, Sousa WM; Redação do manuscrito: Euzebio MB, Vitorino PVO, Sousa WM, Barroso WKS; Revisão crítica do manuscrito quanto ao conteúdo intelectual importante: Euzebio MB, Vitorino PVO, Sousa WM, Sousa ALL, Jardim PCBV, Barroso WKS.

\section{Potencial Conflito de Interesses}

Declaro não haver conflito de interesses pertinentes.

\section{Fontes de Financiamento}

O presente estudo não teve fontes de financiamento externas.

\section{Vinculação Acadêmica}

Este artigo é parte de dissertação de Mestrado de Maicon Borges Euzebio pela Universidade Federal de Goiás

8. Rezende JM, Vitorino PVO, Jardim TSV, Sousa ACS, Jardim PCBV, Souza WKSB. Effects of Long-Term Walking on Baropodometric Parameters and Manual Muscle Strength Journal of Family Medicine. USA: Austin Publishing Group. J Fam Med. 2017;4(3):1116-9.

9. Potter P, Perry A. Fundamentos de Enfermagem. Conceitos, Processo e Prática. Elsevier. 2004; $5^{\mathrm{a}}$ ed.

10. Almeida MFC. Boas Práticas de Laboratório. São Paulo: Difusão Editora; 2008. ISBN: 857808036X.

11. Roche FH-L. [internet]. Procurar informações sobre produtos 2018 [updated 2018 mar 01]. Available from: https://dialog1.roche.com/pt/pt_pt/elabdoc.

12. Lang RM, Badano LP, Mor-Avi V, Jonathan A. Recommendations for Cardiac Chamber Quantification by Echocardiography in Adults: J Am Soc Echocardiogr.2005;28(1):1-34.

13. SOUSA ACS. Avaliação da Função Diastólica do Ventrículo Esquerdo'. In: Carlos Eduardo Suaide Silva. Ecocardiografia - Príncípios e Aplicações Clínicas. 1. 2o ed. Rio de Janeiro: Revinter; 2012. p. 393-415. ISBN-10: 8537204536 .

14. Vicent L, Ariza-Solé A, González-Juanatey J, Uribarri A, Ortiz J, López de Sá E, et al. Exercise-related severe cardiac events. Scand J Med Sci Sports. 2017; 28(4):1404-1411. 
15. Aengevaeren V, Hopman $M$, Thijssen D, van Kimmenade R, de Boer M, Eijsvogels T. Endurance exercise-induced changes in BNP concentrations in cardiovascular patients versus healthy controls. Int J Cardiol. 2017;227(2017):430-5.

16. Legaz-Arrese A, Carranza-García L, Navarro-Orocio R, Valadez-Lira A, Mayolas-Pi C, Munguía-Izquierdo D, et al. Cardiac Biomarker Release after Endurance Exercise in Male and Female Adults and Adolescents.J Pediatr. 2017 Dec 01;91::96-102.

17. Roca E, Nescolarde L, Lupón J, Barallat J, Januzzi J, Liu P, et al. The Dynamics of Cardiovascular Biomarkers in non-Elite Marathon Runners. J Cardiovasc Transl Res. 2017;10(2):206-208

18. Clauss S, Scherr J, Hanley A, Schneider J, Klier I, Lackermair K, et al. Impact of polyphenols on physiological stress and cardiac burden in marathon runners - results from a substudy of the BeMaGIC study. Appl Physiol Nutr Metab. 2017;42(5):523-8

19. Fortescue E, Shin A, Greenes D, Mannix R, Agarwal S, Feldman B, et al. Cardiac troponin increases among runners in the Boston Marathon. Ann Emerg Med. 2007; 49(2):137-43, 143.e1

20. Niemelä M, Kangastupa P, Niemelä O, Bloigu R, Juvonen T. Individual responses in biomarkers of health after marathon and half-marathon running: is age a factor in troponin changes? Scand J Clin Lab Invest. 2016;76(7):575-80

21. Martin T, Pata R, D'Addario J, Yuknis L, Kingston R, Feinn R. Impact of age on haematological markers pre- and post-marathon running. J Sports Sci. 2015;33(19):1988-97.

22. Romagnoli M, Alis R, Aloe R, Salvagno G, Basterra J, Pareja-Galeano H, et al. Influence of training and a maximal exercise test in analytical variability of muscular, hepatic, and cardiovascular biochemical variables. Scand J Clin Lab Invest. 2014;74(3):192-8.

23. Hassan E. Muscle damage and immune responses to prolonged exercise in environmental extreme conditions. J Sports Med Phys Fitness. 2016;56(10):1206-13.

24. Gottschall J, Kram R. Ground reaction forces during downhill and uphill running. J Biomech. 2005;38(3):445-52.

25. Snyder K, Kram R, Gottschall J. The role of elastic energy storage and recovery in downhill and uphill running.J Exp Biol. 2012;215(Pt 13):2283-7.

26. Son H, Lee Y, Chae J, Kim C. Creatine kinase isoenzyme activity during and after an ultra-distance (200 km) run. Biol Sport. 2015;32(4):357-61.

27. Klapcińska B, Waśkiewicz Z, Chrapusta S, Sadowska-Krępa E, Czuba M, Langfort J. Metabolic responses to a 48-h ultra-marathon run in middle-aged male amateur runners. Eur J Appl Physiol. 2013;113(11):2781-93.
28. Eijsvogels T, George K, Shave R, Gaze D, Levine BD, Hopman MT, et al. Effect of prolonged walking on cardiac troponin levels. Am J Cardiol. 2010;105(2):267-72.

29. Sedaghat-Hamedani F, Kayvanpour E, Frankenstein L, Mereles D, Amr A, Buss $\mathrm{S}$, et al. Biomarker changes after strenuous exercise can mimic pulmonary embolism and cardiac injury--a metaanalysis of 45 studies. Clin Chem. 2015;61(10):1246-55.

30. Klinkenberg L, Luyten P, van der Linden N, Urgel K, Snijders D, Knacksted C, et al. Cardiac Troponin T and I Release After a 30-km Run. Am J Cardiol. 2016;118(2):281-7.

31. Chenevier-Gobeaux C, Bonnefoy-Cudraz É, Charpentier S, Dehoux M, Lefevre G, Meune C, et al. High-sensitivity cardiac troponin assays: answers to frequently asked questions. Arch Cardiovasc Dis. 2015;108(2):132-49.

32. Leischik R, Spelsberg N. Endurance sport and "cardiac injury": a prospective study of recreational ironman athletes. Int J Environ Res Public Health. 2014;11(9):9082-100.

33. Nekhanevych O, Zhylyuk V, Logvinenko V, Kramareva Y. The Heart Left Ventricle Diastolic Function During Exercises Of Different Power In Athletes. Georgian Med News. 2017;(262):52-8.

34. Krzemiński K, Buraczewska M, Miśkiewicz Z, Dąbrowski J, Steczkowska $\mathrm{M}$, Kozacz A, et al. Effect of ultra-endurance exercise on left ventricular performance and plasma cytokines in healthy trained men. Biol Sport. 2016;33(1):63-9.

35. Santoro A, Alvino F, Antonelli G, Cassano F, De Vito R, Cameli M, et al. Age related diastolic function in amateur athletes. Int I Cardiovasc Imaging. 2015;31(3):567-73.

36. Sierra A, Ghorayeb N, Dioguardi G, Sierra C, Kiss M. Alteração de biomarcadores de lesão miocárdica em atletas após a Maratona Internacional de São Paulo. Rev Bras Med Sport.. 2015;21(3):182-6.

37. Jouffroy R, Caille V, Perrot S, Vieillard-Baron A, Dubourg O, Mansencal N. Changes of Cardiac Function During Ultradistance Trail Running. Am J Cardiol. 2015;116(8):1284-9.

38. D'Andrea A, Formisano T, Riegler L, Scarafile R, America R, Martone F. Acute and Chronic Response to Exercise in Athletes: The "Supernormal Heart". Adv Exp Med Biol. 2017;999:21-41.

39. Kitagawa $M$, Sugiyama $H$, Morinaga $H$. Serum high-sensitivity cardiac troponin $\mathrm{T}$ is a significant biomarker of left-ventricular diastolic dysfunction in subjects with non-diabetic chronic kidney disease. Nephron extra. 2011;1(1):166-77. 